\title{
COMPLEX STIEFEL MANIFOLDS, SOME HOMOTOPY GROUPS AND VECTOR FIELDS ${ }^{1}$
}

\author{
BY MAURICE E. GILMORE
}

Communicated by William Browder, April 20, 1967

Introduction. Throughout this note $M$ will denote a $\mathfrak{C}^{\infty}$, closed, compact, connected, $2 n$-dimensional, almost-complex manifold. That is, $M$ is an orientable manifold and there is a reduction of the structure group of the tangent bundle of $M$ from $S O(2 n)$ to $U(n)$.

Let real (complex) span $(M)$ denote the maximal number of vector fields on $M$ which are linearly independent over the real (complex) numbers. We obtain certain lower bounds on real (complex) span $(M)$.

Our method is that of obstruction theory in the universal example for such a problem, namely a fibration of the form $W_{n+k, k} \rightarrow B U(n)$ $\rightarrow B U(n+k)$. Here $W_{n+k, k}$ is the Stiefel manifold of complex $k$-frames in complex $(n+k)$-space, and $B U(l)$ is the classifying space for complex $l$-plane bundles. We use the results of Mahowald [2] and Thomas [5] in the obstruction-theoretic problems encountered. The work is done in the category of vector bundles over $c w$-complexes and is then specialized to the case of manifolds.

I extend sincere thanks to my thesis advisor, Emery Thomas, for his aid and encouragement in connection with the work described in this note.

Homotopy groups. We give tables of homotopy groups of $W_{n+k, k}$ useful in doing the obstruction theory. More homotopy groups are given than are actually needed.

It is a classically known fact that $W_{n+k, k}$ is $2 n$-connected. Combining this with the fact that, for $p \leqq 2(k-1)$ a positive integer, $\pi_{2 n+p+1}\left(W_{n+k, k}\right) \approx \pi_{2 n+p+1}\left(W_{n+k+l, k+l}\right)$ for $l \geqq 1$ an integer, we define $\pi_{2 n+p+1}\left(W_{n+k, k}\right)$ to be the $p$-stem of the complex Stiefel manifolds, for $p$ as above. We obtain essentially the first seven of these stems and some results on the 8 and 9-stems. Some unstable results appear in the tables for the sake of completeness. We remark that $W_{n+1,1}=S^{2 n+1}$ and $W_{n, n}=U(n)$ and are not included in the tables, since the homotopy groups in our range of dimensions are well-known for these spaces. The tables list $\pi_{2 n+p+1}\left(W_{n+k, k}\right)$ :

1 These results are part of the author's doctoral thesis submitted to the University of California, Berkeley. 
STIEFEL MANIFOLDS, HOMOTOPY GROUPS AND VECTOR FIELDS
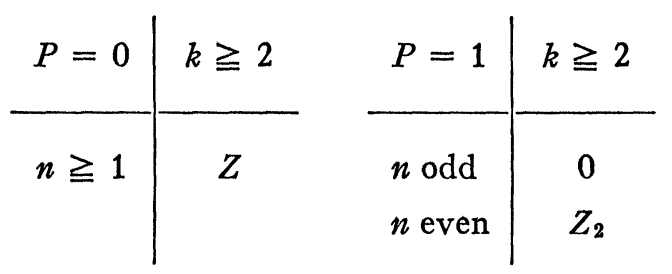

\begin{tabular}{c|c}
$P=2$ & $k \geqq 2$ \\
\hline$n$ odd & $Z$ \\
$n$ even & $Z \oplus Z_{2}$
\end{tabular}

\begin{tabular}{r|cc}
$P=3$ & $k=2$ & $k \geqq 3$ \\
\hline$n=1$ & $Z_{6}$ & 0 \\
2 & $Z_{24} \oplus Z_{2}$ & $Z_{2}$ \\
$n$ odd $\geqq 3$ & $Z_{12}$ & \\
$n$ even $\geqq 4$ & $Z_{24} \oplus Z_{2}$ &
\end{tabular}

and for $k \geqq 3, n \geqq 3$ we have:

$$
\begin{aligned}
& { }^{q} \pi_{2 n+4}\left(W_{n+k, k}\right)=0 \quad \text { for any prime } q \geqq 5 \text {, } \\
& { }^{3} \pi_{2 n+4}\left(W_{n+k, k}\right)=0 \quad \text { if } n \neq \equiv 0(\bmod 3) \text {, } \\
& =Z_{3} \text { if } n \equiv 0(\bmod 3) \text {, } \\
& { }^{2} \pi_{2 n+4}\left(W_{n+k, k}\right)=0 \quad \text { if } n \equiv 3 \text { or } 7(\bmod 8) \\
& =Z_{2} \text { if } n \equiv 1,2 \text { or } 6(\bmod 8) \\
& =Z_{4} \text { if } n \equiv 4 \text { or } 5(\bmod 8) \\
& =Z_{8} \quad \text { if } n \equiv 0(\bmod 8)
\end{aligned}
$$

where ${ }^{q} \pi$ is the $q$-primary part of $\pi$.

\begin{tabular}{r|ll}
$P=4$ & $k=2$ & $k \geqq 3$ \\
\hline$n=1$ & 0 & $Z$ \\
2 & $\left(Z_{2}\right)^{2}$ & $Z \oplus Z_{2}$ \\
$n$ odd $\geqq 3$ & 0 & $Z$ \\
$n$ even $\geqq 4$ & $Z_{2}$ & $Z$
\end{tabular}




\begin{tabular}{r|lll}
\multicolumn{1}{c|}{$P=5$} & \multicolumn{1}{c}{$k=2$} & 3 & $k \geqq 4$ \\
\hline$n=1$ & $Z_{12}$ & $Z_{24}$ & 0 \\
2 & $Z_{24} \oplus Z_{2}$ & $Z_{24}$ & $Z_{3}$ \\
$n$ odd $\geqq 3$ & $Z_{24}$ & $Z_{24} \oplus Z_{2}$ & \\
$n$ even $\geqq 4$ & $Z_{24}$ & $Z_{12}$
\end{tabular}

and, for $n \geqq 3, k \geqq 4$ we have $\pi_{2 n+6}\left(W_{n+k, k}\right) \approx \pi_{2 n+6}\left(W_{n+4,3}\right)$ and is given by the table for $P=3$.

\begin{tabular}{r|clc}
$P=6$ & $k=2$ & 3 & $k \geqq 4$ \\
\hline$n=1$ & $Z_{3}$ & $Z_{2}$ & $Z$ \\
2 & $Z_{2}$ & 0 & $Z$ \\
3 & $Z_{2}$ & $Z_{2}$ & \\
$n \geqq 4$ & $Z_{2}$ & \multicolumn{2}{l}{$Z_{2}$ or 0}
\end{tabular}

and, for $n=3, k \geqq 4$ the group is $Z$ or $Z \oplus Z_{2}$. For $n \geqq 4, k \geqq 4$ we have

$$
\begin{aligned}
& \pi_{2 n+7}\left(W_{n+k, k}\right)=Z \text { if } n \neq 4 s \\
& =Z \text { or } Z \oplus Z_{2} \text { if } n=4 s \text {. } \\
& \begin{array}{l|l}
P=7 & k=2 \\
\hline n=1 & Z_{30} \\
2 & Z_{30} \\
3 & Z_{120} \\
n \geqq 4 & Z_{240}
\end{array} \\
& \begin{array}{r|l}
\multicolumn{1}{c|}{P=8} & \multicolumn{2}{|c}{k=2} \\
n=1 & Z_{4} \\
2 & Z_{2} \oplus Z_{2} \\
3 & Z_{4} \oplus Z_{2} \\
4 & Z_{2} \oplus Z_{2} \oplus Z_{2} \oplus Z_{2} \\
n \text { odd } \geqq 5 & Z_{4} \\
n \text { even } \geqq 6 & Z_{2} \oplus Z_{2} \oplus Z_{2}
\end{array}
\end{aligned}
$$

For the case $P=0$ the group is in Steenrod [4]. In the case $P=3$, $k \geqq 3, n \geqq 3$ the groups follow from the work of Matsunaga [3]. In general, the techniques of Toda, supplemented by information obtained through the use of secondary cohomology operations, give the groups displayed. 
Vector fields. Let $C_{i}(M)$ denote the $i$ th Chern class of the tangent bundle of $M$, where $M$ is a manifold with the properties described above.

Theorem 1. Suppose $H_{2}(M ; Z)$ has no 2-torsion and that $n$ is even, $n \geqq 6$, and $\pi_{1}(M)=0$. Then, if $C_{i}(M)=0$ for $i=n,(n-1)$ and $(n-2)$, real (complex) span $(M) \geqq 6(3)$.

We remark that this theorem was simultaneously proved by Thomas [6] using different methods. We obtain also

CoRollary. Suppose M satisfies the hypotheses of Theorem 1, except that $M$ is not necessarily simply connected. If $n=12 s+2$ or $12 s+10$ then real (complex) span $(M) \geqq 6$ (3).

Proof. The extra obstruction lines in $H^{2 n-1}\left(M ; \pi_{2 n-2}\left(W_{n, 3}\right)\right)$. But it is dead there, since $\pi_{2 n-2}\left(W_{n, 3}\right)=0$, by the above tables in the case $P=3$.

Theorem 2. Suppose $M$ is simply connected, $H_{2}(M ; Z)$ has no torsion, $H_{4}(M ; Z)$ has no 2-torsion, $H_{3}\left(M ; Z_{i}\right)=0$ for $i=2$ or 3 and $n$ is odd, $n \geqq 9$. Then if $C_{i}(M)=0 \quad(n-3) \leqq i \leqq n$, real (complex) span $(M) \geqq 8$ (4).

Theorem 3. Suppose $C_{i}(M)=0$ for $(n-4) \leqq i \leqq n, n$ even, $n \geqq 8$ and $n \neq \equiv 0(\bmod 8)$. Let $\pi_{1}(M)=0=H_{3}(M ; G)=H_{5}(M ; G)$ for $G=Z_{2}$ or $Z_{3}$. Suppose also that $H_{i}(M ; Z)$ has no torsion for $i=2,4$ or 6 . Then real (complex) span $(M) \geqq 10$ (5).

Details of the proofs of these theorems can be found in [1], as well as tables of obstructions. Also larger tables of homotopy groups are given with generators for these groups given in terms of the standard generators for the homotopy groups of spheres.

\section{REFERENCES}

1. M. Gilmore, Homotopy groups of complex Stiefel manifolds, Ph.D. Thesis, University of California, Berkeley, 1967.

2. M. Mahowald, On obstruction theory in orientable fibre bundles, Trans. Amer. Math. Soc. 110 (1964), 315-349.

3. H. Matsunaga, The homotopy groups $\pi_{2 n+i}(U(n))$ for $i=3,4$ and 5, Mem. Fac. Sci., Kyushu Univ., 15 (1960), 72-81. (Note also correction slip.)

4. N. Steenrod, The topology of fibre bundles, Princeton Univ., Press, Princeton, N. J., 1951.

5. E. Thomas, Seminar on fibre spaces, Springer-Verlag, Berlin, 1966.

6. - Postnikov invariants and higher order cohomology operations. Ann. of Math. 85(1967), 184-217.

University of California, Berkeley and NORTHWESTERN UNIVERSITY 